\title{
IDENTIFYING LOGARITHMIC TRACTS
}

\author{
James Waterman \\ The Open University, School of Mathematics and Statistics \\ Walton Hall, Milton Keynes MK7 6AA, United Kingdom; james.waterman@open.ac.uk
}

\begin{abstract}
We show that a direct tract bounded by a simple curve is a logarithmic tract and further give sufficient conditions for a direct tract to contain logarithmic tracts. As an application of these results, it is shown that an example of a function with infinitely many direct singularities, but no logarithmic singularity over any finite value, is in the Eremenko-Lyubich class.
\end{abstract}

\section{Introduction}

Logarithmic tracts, defined below, have found many uses in the iteration of transcendental entire functions. Barański, Karpińska, and Zdunik [2] showed that, if a meromorphic function $f$ has a logarithmic tract, then the dimension of the Julia set of $f$ is strictly greater than 1. Rottenfusser, Rückert, Rempe, and Schleicher [11], as well as Bergweiler, Rippon, and Stallard [4], proved results on the structure of the escaping set for functions with a logarithmic tract. Also, one can construct orbits of points that escape slower than any given sequence within a logarithmic tract [12]. These results use the fact that within a logarithmic tract one can define a logarithmic transform of $f$, which gives an expansion estimate due to Eremenko and Lyubich [6, Lemma 1]. Therefore, it is useful to be able to identify such logarithmic tracts. This motivates the following standard definition of tract from [4] and the rest of this paper.

Definition 1.1. Let $D$ be an unbounded domain in $\mathbf{C}$ whose boundary consists of piecewise smooth curves and suppose that the complement of $D$ is unbounded. Further, let $f$ be a complex valued function whose domain of definition contains the closure $\bar{D}$ of $D$. Then $D$ is called a direct tract of $f$ if $f$ is holomorphic in $D$, continuous in $\bar{D}$, and if there exists $R>0$ such that $|f(z)|=R$ for $z \in \partial D$ while $|f(z)|>R$ for $z \in D$. We call $R$ the boundary value of the direct tract. If, in addition, the restriction $f: D \rightarrow\{z \in \mathbf{C}:|z|>R\}$ is a universal covering, then $D$ is called a logarithmic tract.

Also, if $f^{\prime}(z)=0$ we say $z$ is a critical point and that $f(z)$ is a critical value of $f$. Finally, if $\Gamma:[0, \infty) \rightarrow \mathbf{C}$ with $|\Gamma(t)| \rightarrow \infty$ as $t \rightarrow \infty$ and $f(\Gamma(t)) \rightarrow a$ as $t \rightarrow \infty$, then $a \in \hat{\mathbf{C}}$ is an asymptotic value of $f$ associated with the asymptotic path $\Gamma$.

Every transcendental entire function has a direct tract. Further, the much studied Eremenko-Lyubich class $\mathcal{B}$ consists of those transcendental entire functions for which the set of critical and asymptotic values is bounded. All direct tracts of a function in the class $\mathcal{B}$ are logarithmic tracts, for sufficiently large $R$ in the above.

In this paper, we give a sufficient condition for a direct tract to contain logarithmic tracts. We start with a simple geometric condition for a direct tract to be logarithmic, which perhaps surprisingly does not seem to have been stated previously.

https://doi.org/10.5186/aasfm.2020.4543

2010 Mathematics Subject Classification: Primary 30D05; Secondary 37F10.

Key words: Logarithmic tracts, transcendental entire functions, harmonic functions. 
Theorem 1.1. Let $D$ be a direct tract whose boundary is an unbounded simple curve. Then $D$ is logarithmic.

While direct tracts bounded by a single curve are logarithmic, logarithmic tracts need not be bounded by a single curve. The function $e^{e^{z}}$ has direct tracts which are horizontal strips when the boundary value of the direct tract considered is $R=1$. No critical points lie in these direct tracts and the asymptotic values do not lie in the image of the tracts, so these direct tracts are logarithmic. However, with the additional assumption that there are no asymptotic paths in a logarithmic tract $D$ with finite asymptotic values, then the following converse to Theorem 1.1 is true.

Theorem 1.2. Let $D$ be a logarithmic tract containing no asymptotic paths with finite asymptotic values. Then $D$ is bounded by a single unbounded curve. Further, if $D$ is a logarithmic tract with boundary value $R$, then for all $R^{\prime}>R$, $\left\{z \in D:|f(z)|>R^{\prime}\right\}$ is a logarithmic tract bounded by a simple curve.

Note that, in the first part of the above theorem, $D$ need not be a simple curve. For example, the function $z e^{z}$ with boundary value 1 has a logarithmic tract with a boundary that self intersects.

In the case where a direct tract is bounded by more than one curve, and possibly by infinitely many curves, we give a sufficient condition for the direct tract to contain at least one logarithmic tract. In particular, if a simply connected direct tract is bounded by finitely many curves, we show that the direct tract contains only logarithmic tracts and asymptotic paths with asymptotic values of modulus equal to the boundary value of the direct tract. Further, in the case where there are finitely many boundary curves of a simply connected direct tract, there can be only finitely many critical points in the direct tract, at most $m-1$, where $m$ is the number of logarithmic tracts contained in it. In order to better describe this situation, we define an access to a point, as is done in [1], and a channel of a tract, a new concept based on choosing part of a tract that contains only one access to $\infty$. Note that, only the concept of an access to infinity will be used.

Definition 1.2. Let $U$ be a simply connected domain in C. Fix a point $z_{0} \in U$. A homotopy class of curves $\gamma:[0,1] \rightarrow \hat{\mathbf{C}}$ such that $\gamma([0,1)) \subset U, \gamma(0)=z_{0}$, and $\gamma(t) \rightarrow \infty$ as $t \rightarrow 1$ is called an access from $U$ to $\infty$.

Definition 1.3. Let $D$ be a direct tract. An unbounded simply connected component $G$ of $\{z \in D:|z|>r\}$ for some $r>0$ is called a channel of $D$ if there exists exactly one access to $\infty$ in $G$.

Note that such a channel must be bounded by a single unbounded simple curve.

Theorem 1.3. Let $D$ be a direct tract of $f$, whose boundary includes at least one, and possibly infinitely many, distinct unbounded simple curves, with $|f(z)|=R$, for $z \in \partial D$. Then, for any channel in $D$, either

- the channel contains a logarithmic tract, or

- $f$ has the same finite asymptotic value of modulus $R$ along all paths to infinity in the channel.

Moreover, if $\partial D$ consists of $m$ distinct unbounded simple curves, then $D$ contains at least one logarithmic tract and at most $m-1$ critical points according to multiplicity.

Note that the tract $D$ need not be simply connected in order to satisfy the hypotheses of Theorem 1.3. 
We now recall the classification of singularities of the inverse function due to Iversen [9], using terminology found in [4]. Let $f$ be an entire function and consider $a \in \hat{\mathbf{C}}$. For $r>0$, let $U_{r}$ be a component of $f^{-1}(D(a, r))$ (where $D(a, r)$ is the open disc centered at $a$ with radius $r$ with respect to the spherical metric) chosen so that $r_{1}<r_{2}$ implies that $U_{r_{1}} \subset U_{r_{2}}$. Then either $\bigcap_{r} U_{r}=\{z\}$ for some unique $z \in \mathbf{C}$ or $\bigcap_{r} U_{r}=\emptyset$. These sets $U_{r}$ are called tracts for $f$.

In the first case, we have that $a=f(z)$ and $a$ is an ordinary point if $f^{\prime}(z) \neq 0$, or $a$ is a critical value if $f^{\prime}(z)=0$. In the second case, we have a transcendental singularity over $a$. The transcendental singularity is called direct if there exists $r>0$ such that $f(z) \neq a$ for all $z \in U_{r}$. Otherwise it is indirect. Further, a direct singularity over $a$ is called logarithmic if $f: U_{r} \rightarrow D(a, r) \backslash\{a\}$ is a universal covering.

There are many entire functions with direct tracts which, while not logarithmic, do in fact contain a logarithmic tract, as in Theorem 1.3. This containment corresponds to an access to a logarithmic singularity in a direct tract. Simple examples of this are given in Example 3.1 and Example 3.2. As a more complicated example, Bergweiler and Eremenko [3] constructed an entire function with infinitely many direct, but no logarithmic singularities over any finite value; see Example 3.3. Using Theorem 1.1 we show that Bergweiler and Eremenko's function is in fact in the class $\mathcal{B}$, thus giving an example of a function in the class $\mathcal{B}$ with infinitely many direct singularities, but no logarithmic singularities over any finite value, which was not previously known.

The organization of this paper is the following. Section 2 will be devoted to the proofs of Theorem 1.1, Theorem 1.2, and Theorem 1.3. Section 3 contains three examples to illustrate applications of these results.

Remark (added in proof). The proofs of all the results about direct tracts in this paper have made the tacit assumption that any unbounded piecewise smooth curve in the boundary tends to $\infty$ at both ends; this would be the case for a direct tract of a transcendental meromorphic function, and for any subtract $\left\{z:|f(z)|>R^{\prime}\right\}$, for $R^{\prime}>R$, of a general direct tract with boundary value $R$. The author is grateful to Prof. James Langley for pointing out the theoretical existence of direct tracts whose boundaries include unbounded smooth curves that accumulate at finite boundary points, though we do not know of any examples of such tracts.

\section{Proofs of Theorem 1.1, Theorem 1.2, and Theorem 1.3}

The proof of Theorem 1.1, which states that direct tracts bounded by a simple curve are logarithmic, is straightforward and similar to the proof of [3, Theorem 5], though the statement of [3, Theorem 5] is somewhat different. We include this proof for completeness.

Proof of Theorem 1.1. Let $\phi: \mathbf{D} \rightarrow D$ be a Riemann map, where $\mathbf{D}$ denotes the open unit disc. The following construction is illustrated in Figure 1. The set $D$ is a Jordan domain in the Riemann sphere with boundary $\partial D \cup\{\infty\}$, so $\phi$ extends continuously and one-to-one to $\partial \mathbf{D}$, by Carathéodory's Theorem ([5] and [7, Theorem I.3.1]), and without loss of generality $\phi(1)=\infty$. So,

$$
u(t)=\log \frac{|f(\phi(t))|}{R}, \quad \text { for } t \in \mathbf{D},
$$

is a positive harmonic function in $\mathbf{D}$ with $u(t)=0$ if $t \in \partial \mathbf{D} \backslash\{1\}$. Therefore $u$ is a positive multiple of the Poisson kernel in $\mathbf{D}$ with singularity at 1 . For a discussion of 


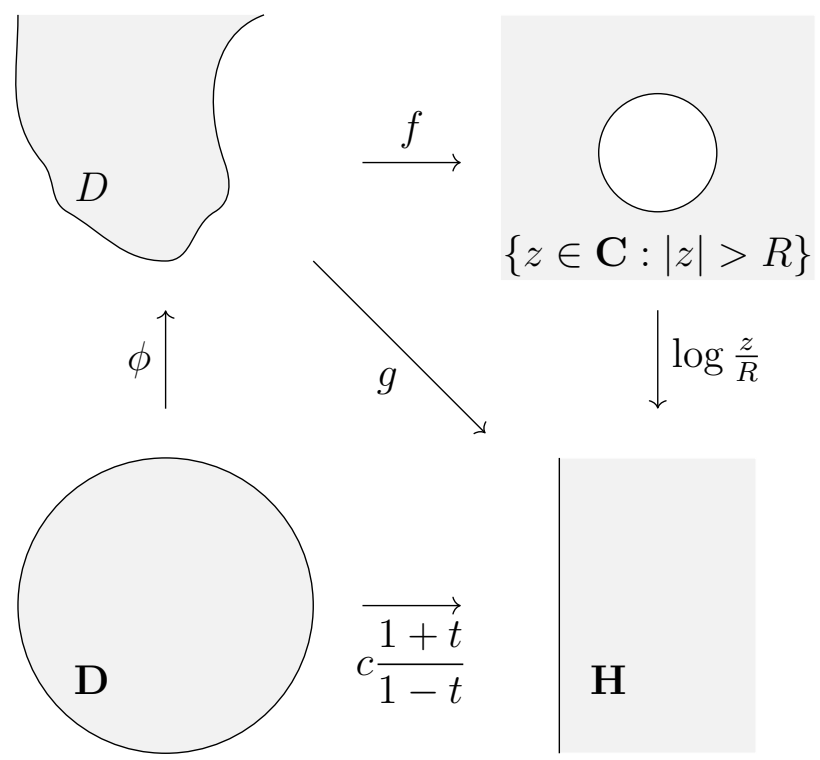

Figure 1. Construction in the proof of Theorem 1.1.

positive harmonic functions, and the Poisson kernel and its properties, see [7] or [8], for example. Hence,

$$
u(t)=c \operatorname{Re}\left(\frac{1+t}{1-t}\right), \quad \text { where } c>0 .
$$

Now we can define an analytic branch $g$ of $\log f / R$ in $D$ by the monodromy theorem, since $D$ is simply connected and any local branch $g$ of $\log f / R$ can be analytically continued along any path in $D$. Then,

$$
g(\phi(t))=\log \frac{f(\phi(t))}{R}, \quad \text { for } t \in \mathbf{D}
$$

is analytic in $\mathbf{D}$, with

$$
\operatorname{Re} g(\phi(t))=\log \frac{|f(\phi(t))|}{R}=c \operatorname{Re}\left(\frac{1+t}{1-t}\right) .
$$

Hence, for some constant $\theta \in \mathbf{R}$, we have

$$
\begin{aligned}
g(\phi(t))=c \frac{1+t}{1-t}+i \theta & \Longrightarrow f(\phi(t))=R e^{i \theta} \exp \left(c \frac{1+t}{1-t}\right), \quad \text { for } t \in \mathbf{D}, \\
& \Longrightarrow f(z)=\operatorname{Re}^{i \theta} \exp \left(c \frac{1+\phi^{-1}(z)}{1-\phi^{-1}(z)}\right), \quad \text { for } z \in D .
\end{aligned}
$$

It follows immediately that $f$ has no critical points in $D$. Also, there are no asymptotic paths in $D$ with finite asymptotic values, as the exponential function has none in $\mathbf{H}=\{z: \operatorname{Re} z>0\}$. Indeed, if $\gamma \rightarrow \infty$ in $D$, then $\phi^{-1}(z) \rightarrow 1$ along $\gamma$. So, $\left(1+\phi^{-1}(z)\right) /\left(1-\phi^{-1}(z)\right) \rightarrow \infty$ in $\mathbf{H}$ as $z \rightarrow \infty$ for $z \in \gamma$. Hence, by (1), $f(z)$ cannot tend to a finite limit as $z \rightarrow \infty$ for $z \in \gamma$.

Now, we prove Theorem 1.2, giving a converse to Theorem 1.1.

Proof of Theorem 1.2. Let $D$ be a logarithmic tract with boundary value $R$. Then the function $g(z)=\log f(z)$ is a univalent map from $D$ onto $H_{R}=\{t: \operatorname{Re} t>\log R\}$, with a univalent inverse function $h: H_{R} \rightarrow D$.

Now, $D$ is simply connected and $\partial D$ consists of piecewise smooth curves. Thus, on the Riemann sphere, the boundary of $D$ is the curve $\partial D \cup\{\infty\}$ (not necessarily 
a simple curve). Therefore, by [10, Theorem 2.1], the univalent function $h$ extends continuously to $\partial H_{R} \cup\{\infty\}$, with values in $\partial D \cup\{\infty\}$. If there exists $t_{0} \in \partial H_{R}$ such that $h\left(t_{0}\right)=\infty$, then the function $g=h^{-1}$ has asymptotic value $t_{0}$ in $D$. So, by our assumption, $h(t)$ must be finite for all $t \in \partial H_{R}$. Hence, $h\left(\partial H_{R}\right)=\partial D$ is a single unbounded curve, possibly with self-intersection.

The final part of the theorem follows immediately from the above discussion of $g$ and $h$, since

$$
\left\{z \in D:|f(z)|=R^{\prime}\right\}=h\left(L_{R^{\prime}}\right),
$$

where $L_{R^{\prime}}=\left\{t: \operatorname{Re} t=\log R^{\prime}\right\}$ and $R^{\prime}>R$.

The proof of Theorem 1.3 uses similar machinery, but is rather more complicated.

Proof of Theorem 1.3. Consider a direct tract $D$ bounded by more than one unbounded curve with $|f(z)|=R$, for $z \in \partial D$. If it exists, choose some channel, $G$ of $D$. Then either $|f(z)|$ will be unbounded or bounded within this channel. Let $\phi: \mathbf{D} \rightarrow G$ be a Riemann map with $\phi(z) \rightarrow \infty$ as $z \rightarrow 1$ for $z \in \mathbf{D}$. This is possible by the definition of a channel. Then, $\phi$ extends continuously and one-to-one to $\partial \mathbf{D}$ by Carathéodory's Theorem, and once again $\phi(1)=\infty$.

Let $E$ be the subset of $\partial G$ where $|f(z)| \neq R$. Then,

$$
u(t)=\log \frac{|f(\phi(t))|}{R}, \quad \text { for } t \in \mathbf{D}
$$

is a positive harmonic function in $\mathbf{D}$. Also, the set $\phi^{-1}(E)$ is contained in a closed arc of $\partial \mathbf{D}$ which does not contain 1 . Note that $\phi^{-1}(E)$ may be disconnected.

First, assume $f$ is unbounded in $G$ and denote by $P(t, \zeta)$ the Poisson kernel in $\mathbf{D}$ with singularity at $\zeta$. Since $f$ is unbounded in $G$, it follows from the Poisson integral formula (see [8, Theorem 1.16]) that

$$
u(t)=c P(t, 1)+\int_{\phi^{-1}(E)} P(t, \zeta) u(\zeta) d \lambda(\zeta), \quad \text { for } t \in \mathbf{D}
$$

where $c>0$ and $\lambda(\zeta)$ is the normalized Lebesgue measure on $\partial \mathbf{D}$. Now, choose $\epsilon>0$ and $\delta>0$ such that

$$
\int_{\phi^{-1}(E)} P(t, \zeta) u(\zeta) d \lambda(\zeta)<\epsilon
$$

for $|t-1|<\delta$ and $t \in \mathbf{D}$. Then, choose $R_{2}>R_{1}>0$ such that the horodiscs

$$
H_{j}=\left\{t \in \mathbf{D}: c P(t, 1)>R_{j}\right\}, \quad \text { for } j=1,2,
$$

lie inside $D(1, \delta)$ and $R_{2}>R_{1}+2 \epsilon$. So, for $t \in H_{2}, u(t)>c P(t, 1)>R_{2}$. Let $\Omega$ be the component of $\left\{t \in \mathbf{D}: u(t)>R_{2}\right\}$ that contains $H_{2}$. For $t \in \Omega, u(t)>R_{2}$, so

$$
c P(t, 1)>R_{2}-\int_{\phi^{-1}(E)} P(t, \zeta) u(\zeta) d \lambda(\zeta) .
$$

Hence, by (2) and (3), $c P(t, 1)>R_{2}-\epsilon>R_{1}$, for $t \in \Omega$. Therefore, $H_{2} \subset \Omega \subset H_{1}$, as shown in Figure 2. 


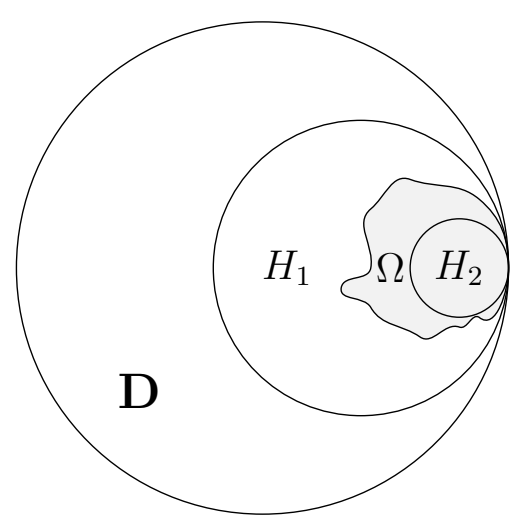

Figure 2. $H_{2} \subset \Omega \subset H_{1} \subset \mathbf{D}$.

We next claim that $\Omega$ is bounded by a single curve if $R_{2}$ is sufficiently large. To prove this, consider the Riemann map $\psi: \mathbf{D} \rightarrow \mathbf{H}$ given by $\psi(t)=\frac{1+t}{1-t}$. Then, consider the positive harmonic function $U$ on $\mathbf{H}$ defined by

$$
\begin{aligned}
U(x+i y) & =u\left(\psi^{-1}(x+i y)\right) \\
& =c P\left(\psi^{-1}(x+i y), 1\right)+\int_{\psi\left(\phi^{-1}(E)\right)} P\left(\psi^{-1}(x+i y), \psi^{-1}(s)\right) u\left(\psi^{-1}(s)\right) \frac{d s}{1+s^{2}} \\
& =c x+x \int_{\psi\left(\phi^{-1}(E)\right)} \frac{U(s) d s}{x^{2}+(y-s)^{2}}, \quad \text { for } x+i y \in \mathbf{H} .
\end{aligned}
$$

We have

$$
\begin{aligned}
\frac{\partial U(x+i y)}{\partial x} & =c+\int_{\psi\left(\phi^{-1}(E)\right)} \frac{U(s) d s}{x^{2}+(y-s)^{2}}+x \frac{\partial}{\partial x} \int_{\psi\left(\phi^{-1}(E)\right)} \frac{U(s) d s}{x^{2}+(y-s)^{2}} \\
& \geq c-\int_{\psi\left(\phi^{-1}(E)\right)} \frac{2 x^{2} U(s)}{\left(x^{2}+(y-s)^{2}\right)^{2}} d s \\
& \geq c-\frac{2}{x^{2}} \int_{\psi\left(\phi^{-1}(E)\right)} U(s) d s>0
\end{aligned}
$$

for $x$ sufficiently large and $y \in \mathbf{R}$, since $\psi\left(\phi^{-1}(E)\right)$ is contained in a bounded interval of the imaginary axis and $U(s)$ is bounded on $\psi\left(\phi^{-1}(E)\right)$. So, $\frac{\partial U(x+i y)}{\partial x}>0$ in the halfplane $\psi\left(H_{1}\right)$ for sufficiently small $\delta$. Therefore, $U(x+i y)$ is monotonic with respect to $x$ in the half-plane $\psi\left(H_{1}\right)$ for any fixed $y$. Hence $\left\{x+i y: U(x+i y)>R_{2}\right\}$ is bounded by a single simple unbounded curve, and so $\Omega=\psi^{-1}\left(\left\{x+i y: U(x+i y)>R_{2}\right\}\right)$ is a Jordan domain bounded by a simple curve. Thus, by Theorem 1.1, $\phi(\Omega)$ is a logarithmic tract in $G$.

Now, assume that $f$ is bounded in $G$. Then there exists $K$ such that $u(t)<K$ for $t$ in a neighborhood of the boundary singularity at 1 . On the boundary of $\mathbf{D}, u(t) \equiv 0$ for $t$ in a neighborhood of 1 except possibly at 1 . So, by the extended maximum principle [8, Theorem 5.16], $u$ has boundary value 0 at 1 . We want to show that $f(\phi(t)) \rightarrow \alpha$ as $t \rightarrow 1$, where $|\alpha|=R$. Since $u \equiv 0$ on $\partial \mathbf{D}$ in a neighborhood of 1 , we deduce, by the reflection principle [8, Example 1, p. 35], that we can find a neighborhood, $N$ say, of 1 in $\mathbf{C}$ to which $u$ extends harmonically. Therefore, there exists a complex conjugate $v$ of $u$ so that $u+i v$ is analytic on this neighborhood. Let

$$
g(z)=R \exp \left(u\left(\phi^{-1}(z)\right)+i v\left(\phi^{-1}(z)\right)\right), \quad \text { for } z \in \phi(N \cap \mathbf{D}) .
$$

Then, $g$ is analytic on $\phi(N \cap \mathbf{D}) \subset G$ and $|g(z)|=|f(z)|$ in $\phi(N \cap \mathbf{D})$. Non-constant analytic maps are open, so $g(z)=c f(z)$ in $\phi(N \cap \mathbf{D})$, where $|c|=1$. Therefore, 
$\arg f(z)$ tends to a finite limit as $z \rightarrow \infty$ in $\phi(N \cap \mathbf{D})$, and hence in $G$, since any sequence tending to infinity in $G$ is eventually contained in $\phi(N \cap \mathbf{D})$. Therefore, there exists $\alpha$ such that $f(z) \rightarrow \alpha$ as $z \rightarrow \infty$ in $G$, with $|\alpha|=R$.

Finally, if $D$ is bounded by $m$ distinct unbounded simple curves, then following the method in the proof of Theorem 1.1, we again define the positive harmonic function

$$
u(t)=\log \frac{|f(\phi(t))|}{R}, \quad \text { for } t \in \mathbf{D},
$$

where $\phi: \mathbf{D} \rightarrow D$ is a conformal map and each access to infinity in $D$ corresponds under $\phi$ to a family of paths tending to a point on $\partial \mathbf{D}$ along which $u$ tends to either 0 or $\infty$. Suppose there exist $n \leq m$ accesses to infinity on which $f$ is unbounded. Then, there exist $n$ points $\zeta_{1}, \ldots, \zeta_{n} \in \partial \mathbf{D}$ such that $u$ is a positive harmonic function in $\mathbf{D}$ with $u(t)=0$, for $t \in \partial \mathbf{D} \backslash\left\{\zeta_{1}, \ldots, \zeta_{n}\right\}$. Therefore $u$ is a sum of positive multiples of the Poisson kernel in $\mathbf{D}$ with singularities at $\zeta_{1}, \ldots, \zeta_{n}$. Hence,

$$
u(t)=\sum_{k=1}^{n} c_{k} \operatorname{Re}\left(\frac{\zeta_{k}+t}{\zeta_{k}-t}\right), \quad \text { where } c_{k}>0, \text { for } k=1, \ldots, n .
$$

Now, again, we can define an analytic branch $g$ of $\log f / R$ in $D$ by the monodromy theorem, since $D$ is simply connected and any local branch $g$ of $\log f / R$ can be analytically continued along any path in $D$. Then,

$$
g(\phi(t))=\log \frac{f(\phi(t))}{R}, \quad \text { for } t \in \mathbf{D}
$$

is analytic in $\mathbf{D}$, with

$$
\operatorname{Re} g(\phi(t))=\log \frac{|f(\phi(t))|}{R}=\sum_{k=1}^{n} c_{k} \operatorname{Re}\left(\frac{\zeta_{k}+t}{\zeta_{k}-t}\right) .
$$

Hence, for some constant $\theta \in \mathbf{R}$, we have

$$
g(\phi(t))=\sum_{k=1}^{n} c_{k} \frac{\zeta_{k}+t}{\zeta_{k}-t}+i \theta \Longrightarrow f(\phi(t))=R e^{i \theta} \exp \left(\sum_{k=1}^{n} c_{k} \frac{\zeta_{k}+t}{\zeta_{k}-t}\right), \quad \text { for } t \in \mathbf{D} .
$$

The critical points of $f(\phi(t))$ for $t \in \mathbf{D}$ are the solutions of

$$
\sum_{k=1}^{n} c_{k} \frac{2 \zeta_{k}}{\left(\zeta_{k}-t\right)^{2}}=0
$$

for which there are at most $2 n-2$ solutions in C. Further, by the Cauchy-Riemann equations, critical points of $f(\phi(t))$ occur if and only if $|\nabla u(t)|=0$, where $\nabla u$ denotes the gradient of $u$. For a point $t \in \mathbf{D}$, noting that $\zeta_{k}=1 / \bar{\zeta}_{k}$ gives that

$$
\operatorname{Re}\left(\frac{\zeta_{k}+t}{\zeta_{k}-t}\right)=-\operatorname{Re}\left(\frac{\zeta_{k}+1 / \bar{t}}{\zeta_{k}-1 / \bar{t}}\right), \quad \text { for } k=1, \ldots, n .
$$

Hence, each Poisson kernel is 'symmetric' under reflection in the unit circle and so a sum of Poisson kernels is as well. Further, no solutions of $|\nabla u(t)|=0$ lie on $\partial \mathbf{D}$, by the behavior of the Poisson kernel near $\partial \mathbf{D}$. So, the solutions of $|\nabla u(t)|=0$ occur in pairs and are symmetric with respect to $\partial \mathbf{D}$. Therefore, $\mathbf{D}$ contains at most $n-1$ critical points of $f(\phi(t))$ and hence $D$ contains at most $n-1$ critical points of $f$. 


\section{Examples}

In this section, we give three examples to show the kinds of direct tracts that can exist, and to which we can apply Theorem 1.1 and Theorem 1.3. First, to illustrate Theorem 1.3, we give a simple example of a transcendental entire function with a simply connected direct tract bounded by finitely many boundary curves.

Example 3.1. Consider $G(z)=2 \exp \left(z^{4}\right)$. Note that $G$ is in the class $\mathcal{B}$.

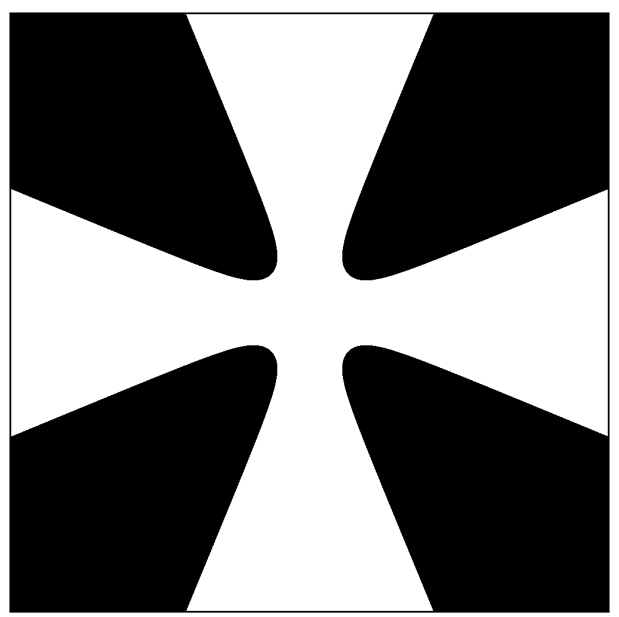

Figure 3. The tract of $2 \exp \left(z^{4}\right)$ in white with its complement in black.

First, $G(z) \rightarrow \infty$ as $z \rightarrow \infty$ along the real and imaginary axes, and $G(z) \rightarrow 0$ as $z \rightarrow \infty$ along the rays with angle an odd multiple of $\pi / 4$. With a boundary value of $R=1$, as in Figure 3, a neighborhood about the origin is contained in the direct tract. Further, on the lines with angle an odd multiple of $\pi / 8$ the modulus of $G$ is 2. Hence, $G$ has one direct tract bounded by four unbounded simple curves. By Theorem 1.3, $G$ contains at most three critical points, and in fact, contains a single critical point of multiplicity 3 at 0 . The direct tract of $G$ has four channels on which $f$ is unbounded and hence contains four logarithmic tracts.

Next, we illustrate Theorem 1.3 by giving an example of a direct non-logarithmic tract with infinitely many logarithmic tracts inside it.

Example 3.2. Consider $f(z)=\exp (\sin (z)-z)$. Note that $f$ is not in the class $\mathcal{B}$ as it has critical values at $e^{2 k \pi}$ for all $k \in \mathbf{Z}$.

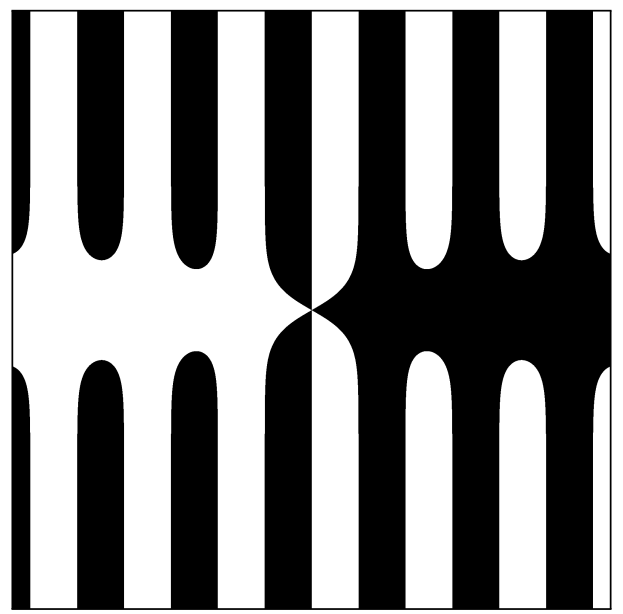

Figure 4. The tracts of $\exp (\sin (z)-z)$ in white with their complement in black. 
First, $f(z) \rightarrow \infty$ as $z \rightarrow \infty$ along the negative real axis and $f(z) \rightarrow \infty$ as $z \rightarrow \infty$ along translates of the imaginary axis by $\frac{\pi}{2}+2 k \pi$, for $k \in \mathbf{Z}$. Also, $f(z) \rightarrow 0$ as $z \rightarrow \infty$ along the positive real axis and along translates of the imaginary axis by $\frac{3 \pi}{2}+2 k \pi$, for $k \in \mathbf{Z}$. So, $f$ will have infinitely many direct tracts in the right half-plane and one direct tract in the left half-plane, with $|f(z)|=R$ on $\partial D$ for some suitable $R>0$. See Figure 4 , where $R=1$. Further, $f$ has no zeros and its only finite asymptotic value is 0 , along the positive real axis and translates of the imaginary axis by $\frac{3 \pi}{2}+2 k \pi$, for $k \in \mathbf{Z}$. Hence, by Theorem 1.3 , the direct tract in the left half-plane contains infinitely many logarithmic tracts, each corresponding to channels about translates of the positive and negative imaginary axes by $\frac{\pi}{2}+2 k \pi$, for $k \in \mathbf{Z}$. In contrast, the tracts in the right half-plane are themselves logarithmic tracts in their own right.

Finally, we use our results to show that an entire function constructed by Bergweiler and Eremenko [3] to have no logarithmic singularities over any finite value is, moreover, in the class $\mathcal{B}$. Note that the reciprocal of this function is an example of a function with a direct tract which, while simply connected, does not contain any channels.

Example 3.3. Consider the entire function

$$
h(z)=\exp (g(z)), \quad \text { where } g(z)=\sum_{k=1}^{\infty}\left(\frac{z}{2^{k}}\right)^{2^{k}} .
$$

Then, $h$ has infinitely many direct singularities, but no logarithmic singularity over any finite value, and is in the class $\mathcal{B}$.

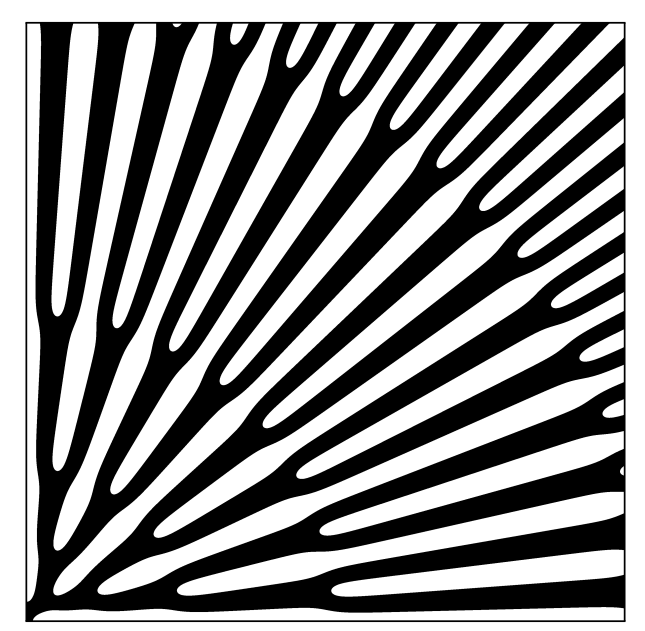

Figure 5. The direct tracts of $h$ over $\infty$ in white with the direct tract over 0 in black, for $0 \leq \operatorname{Re} z \leq 250$ and $0 \leq \operatorname{Im} z \leq 250$.

The first two statements are proved in [3], so it remains to check that $h$ is in the class $\mathcal{B}$. In order to see this, we will show that every direct tract (over $\infty$ ) for some fixed boundary value is bounded by a single curve, and so all the direct tracts are logarithmic by Theorem 1.1. This implies that no critical points lie in these tracts and no asymptotic paths for finite asymptotic values, and so the singular values of $h$ form a bounded set. We then conclude that $h$ is in the class $\mathcal{B}$.

Following the notation and construction in [3], fix $\varepsilon$ with $0<\varepsilon \leq \frac{1}{8}$ and set $r_{n}=(1+\varepsilon) 2^{n+1}$ and $r_{n}^{\prime}=(1-2 \varepsilon) 2^{n+2}$ for $n \in \mathbf{N}$. Then for $j \in\left\{0,1, \cdots, 2^{n}-1\right\}$ 
we define the sets

and

$$
B_{j, n}=\left\{r \exp \left(\frac{\pi i}{2^{n}}+\frac{2 \pi i j}{2^{n}}\right): r_{n} \leq r \leq r_{n}^{\prime}\right\}
$$

$$
C_{j, n}^{ \pm}=\left\{r \exp \left(\frac{\pi i}{2^{n}}+\frac{2 \pi i j}{2^{n}} \pm \frac{r-r_{n}^{\prime}}{r_{n+1}-r_{n}^{\prime}} \frac{\pi i}{2^{n+1}}\right): r_{n}^{\prime} \leq r \leq r_{n+1}\right\} .
$$

Bergweiler and Eremenko [3, Section 6] show that every unbounded simple path starting at 0 and lying in the infinite tree,

$$
T=\left[-i r_{1}, i r_{1}\right] \cup \bigcup_{n=1}^{\infty} \bigcup_{j=1}^{2^{n}-1}\left(B_{j, n} \cup C_{j, n}^{ \pm}\right),
$$

is an asymptotic curve along which the function tends to 0. Further,

$$
\operatorname{Re} g(z)<-2^{2^{n}} \quad \text { for } z \in B_{j, n} \cup C_{j, n}^{ \pm} .
$$

So, if some direct tract (over $\infty$ ) with a sufficiently large boundary value was not bounded by a single curve, then at least one of the following three possibilities would occur:

(1) there would be another direct tract over a finite value,

(2) the tract would have to cross the infinite tree $T$,

(3) there would be zeros of the function $h$.

The first case is shown not to happen in [3, Section 6] by proving that $\arg g\left(r e^{i \theta}\right)$ is an increasing function of $\theta$, that it increases by $2^{n} 2 \pi$ as $\theta$ increases by $2 \pi$, and then using a counting argument to show that all the direct tracts are accounted for and there can be no others. We can assume that the tract does not cross the infinite tree $T$, since $h(z)$ is bounded on $T$ by a value smaller than the tract boundary value. Finally, the last case cannot happen since the exponential function omits 0 . Therefore, each direct tract (over $\infty$ ) is bounded by a single curve, so by Theorem 1.1 we can deduce that $h \in \mathcal{B}$.

Acknowledgements. I would like to thank my supervisors Prof. Phil Rippon and Prof. Gwyneth Stallard for their guidance and encouragement in the preparation of this paper. I would also like to thank Dr. Dave Sixsmith for mentioning the problem of finding an example of a function in the class $\mathcal{B}$ with a direct non-logarithmic singularity over a finite value, which led to Example 3.3. I would finally like to thank the referees for many helpful comments and suggestions.

\section{References}

[1] Barański, K., N. Fagella, X. Jarque, and B. Karpińska: Accesses to infinity from Fatou components. - Trans. Amer. Math. Soc. 369:3, 2017, 1835-1867.

[2] Barański, K., B. Karpińska, and A. Zdunik: Hyperbolic dimension of Julia sets of meromorphic maps with logarithmic tracts. - Int. Math. Res. Not. IMRN 2009:4, 2009, 615-624.

[3] Bergweiler, W., and A. Eremenko: Direct singularities and completely invariant domains of entire functions. - Illinois J. Math. 52:1, 2008, 243-259.

[4] Bergweiler, W., P. J. Rippon, and G. M. Stallard: Dynamics of meromorphic functions with direct or logarithmic singularities. - Proc. Lond. Math. Soc. (3) 97:2, 2008, 368-400.

[5] Collingwood, E. F., and A. J. Lohwater: The theory of cluster sets. - Cambridge Tracts in Math. and Math. Phys. 56, Cambridge Univ. Press, Cambridge, 1966.

[6] Eremenko, A. E., and M. Yu. Lyubich: Dynamical properties of some classes of entire functions. - Ann. Inst. Fourier (Grenoble) 42:4, 1992, 989-1020. 
[7] Garnett, J. B., and D. E. Marshall: Harmonic measure. - New Math. Monogr. 2, Cambridge Univ. Press, Cambridge, 2005.

[8] Hayman, W. K., and P. B. Kennedy: Subharmonic functions. Volume I. - London Math. Soc. Monogr. Ser. 9, Academic Press, Harcourt Brace Jovanovich, Publishers, London-New York, 1976.

[9] Iversen, F.: Recherches sur les fonctions inverses des fonctions méromophes. - PhD thesis, Helsingfors, 1914.

[10] Pommerenke, Ch.: Boundary behaviour of conformal maps. - Grundlehren Math. Wiss. 299, Springer-Verlag, Berlin, 1992.

[11] Rottenfusser, G., J. Rückert, L. Rempe, and D. Schleicher: Dynamic rays of boundedtype entire functions. - Ann. of Math. (2) 173:1, 2011, 77-125.

[12] Waterman, J.: Slow escape in tracts. - Proc. Amer. Math. Soc. 147:7, 2019, 3087-3101.

Received 14 March 2019 • Accepted 13 August 2019 